\title{
Depth of Clinical Pharmacology in Undergraduate Medical Education
}

Frederick J. Goldstein, PhD

Financial Disclosures: None reported. Support: None reported. Address correspondence to Frederick J. Goldstein, PhD, Department of Biomedical Sciences, Philadelphia College of Osteopathic Medicine, 4170 City Ave, Philadelphia, PA, 19131-1610.

Email: fredg@pcom.edu

Submitted July 30, 2018; revision received October 3, 2018;

accepted October 31, 2018.
$\mathrm{W}$ here were you on February 5, 1968? Some osteopathic physicians were not yet born, and others were in early education or osteopathic medical school. On that day, I was awarded my doctorate in pharmacology and began my career as a full-time professional in health care.

After earning my doctorate, I began teaching courses on the principles of clinical pharmacology as well as on the majority of neuropharmacologic agents at the University of the Sciences in Philadelphia, Pennsylvania. At that time, there was a relatively limited quantity of such agents.

However, over my 5 decades in this professional endeavor (of which the past 26 years have been as professor of clinical pharmacology at the Philadelphia College of Osteopathic Medicine [PCOM]), there has been an explosion in the number of available pharmacologic agents. According to Kinch et al, ${ }^{1}$ there were about 400 FDA-approved new molecular entities in 1968 compared with 1400 in 2013 - approximately a 350\% increase (Figure). Although some of these entities have been recalled, the vast majority remain available for prescribing.

I review articles every day for categories of agents I teach (eg, antidepressants, antipsychotics, opioids). Most neuropharmalogic medications were not even available in 1968 (eg, medications for managing multiple sclerosis, monoclonal antibodies, Janus kinase inhibitors). I determine how to best present neuropharmacology to PCOM's first- and second-year students. How much drug information - especially regarding mechanisms of action-does a physician need to know to care for patients at the highest level? In my opinion, there is a limit, but it does, of course, depend on the category. Is it really important for a doctor to identify exactly what G-protein is affected for a medication that is being prescribed? For some conditions, it could be. For most, not really.

This question of how much data should be presented in medical education is not new. An excerpt from a 1944 report follows:

There is an urgent need in every medical school for a new viewpoint on the part of those who teach pre-clinical subjects to drastically eliminate from the curricula, and therefore from tests in these subjects, a mass of detailed information which serves only to clutter students' minds and deaden their interest in topics that provide the liveliest appeal to them. ${ }^{2}$

There are clear examples when subcellular information is clinically relevant to understand the action of medications. Examples include agents that block calcium channels (eg, nifedipine), impair the release of this ion (eg, dantrolene), or facilitate the influx of chloride (eg, diazepam). Morphine also inhibits the release of calcium, knowledge of which helps to explain why nifedipine can increase analgesic efficacy of this opioid.

When I started teaching, subtypes of dopamine receptors D1 and D2, major aspects of the mechanism of action of antipsychotics, were about to be discovered. Potentially severe consequences could occur if the wrong agonist or antagonist is prescribed. Knowing that newer atypical agents (eg, olanzapine) are much less likely to cause 


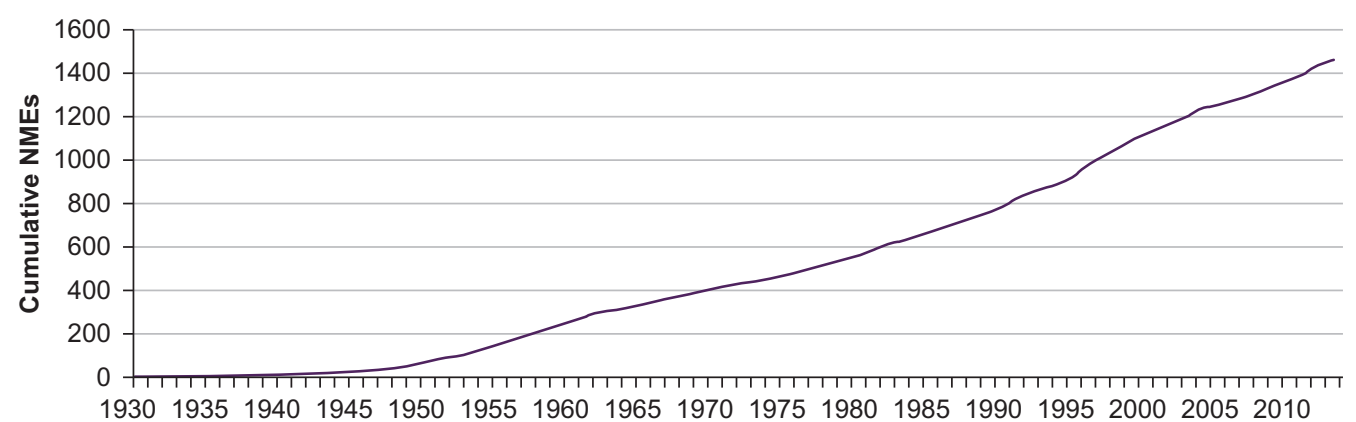

Figure

Reprinted with permission. Source: Kinch MS, Haynesworth A, Kinch SL, Hoyer D. An overview of FDA-approved new molecular entities: 1827-2013. Drug Discov Today. 2014;19(8):1033-1039. doi:10.1016/j.drudis.2014.03.018.

tardive dyskinesia because they act differently on D1 and D2 receptors than typical agents (eg, haloperidol) is a key aspect of prescribing these drugs. However, do most prescribers need to know that the D2 receptor is coded on chromosome 11 , reduces cyclic adenosine monophosphate via Gi-coupled inhibition of adenylyl cyclase, and more? Except for those physicians engaged in molecular pharmacologic research, does it make sense to present these finite aspects at the subcellular level to future physicians? I say no.

\section{Osteopathic vs Allopathic Licensing Examinations}

By July 2020, all osteopathic and allopathic graduates will be training in programs accredited by the Accreditation Council of Graduate Medical Education. ${ }^{3}$ Although osteopathic medical students are expected to complete the Comprehensive Osteopathic Medical Licensing Examination-USA (COMLEXUSA) series, feedback that I've received from students clearly indicates that certain specialties still require osteopathic medical students to take the United States Medical Licensing Examination (USMLE) to be considered for admission.

This situation thus presents a dilemma, and as a coordinator of pharmacology, I need to know about general differences between the examinations. Many medical students who have taken both the
COMLEX-USA and the USMLE have conveyed to me that the latter delves deeply into the subcellular world to a much greater degree than the former. In my opinion, that does not mean that "their" system is better than "our" system. As a member of the National Board of Osteopathic Medical Examiners (the organization that creates COMLEX-USA) for many years, I firmly believe that COMLEX-USA is a more realistic clinical examination than the USMLE.

How to solve this problem? Considering that the amount of time used for presenting material is the same as it has been for more than a century in medical education, the only solution I see is to place more information online so that students can study it at their own pace. Faculty may not even have to put that information in electronic format because such data are contained in top-level pharmacology textbooks, some of which are available online. Ultimately, however, students will have to learn this information on their own. This burden is increased for osteopathic medical students, who have many scheduled hours for osteopathic manipulative medicine education (currently 165 hours at PCOM in the first 2 years); allopathic medical students have no such additional time commitment.

\section{Conclusion}

As noted by the London General Medical Council in 1957: 
In regard to medical education, there is a need to reduce the sheer factual load on students, and to ensure that the memorizing and reproduction of factual data are not allowed to interfere with the primary need for fostering critical study of principles and the development of independent thoughts. ${ }^{4}$

Those of us who teach should work to preserve our students' mental health to reduce their chances of burning out. A focus on presenting information directly relevant to the practice of medicine is important to achieve this goal. (doi:10.7556/jaoa.2019.104)

\section{References}

1. Kinch MS, Haynesworth A, Kinch SL, Hoyer D. An overview of FDA-approved new molecular entities: 1827-2013. Drug Discov Today. 2014;19(8):1033-1039. doi:10.1016/j.drudis.2014.03.018

2. Goodenough W. Report of the Inter-departmental Committee on Medical Schools. S.O. Code 32-363. London H.M. Stationery Office; 1944. Reprinted in: Simpson MA. Medical Education: A Critical Approach. London, UK: Butterworth and Company; 1972:A13.

3. Buser BR, Swartwout J, Lischka T, Biszewski M. Single accreditation system for graduate medical education: transition update. J Am Osteopath Assoc. 2019;119(4):257-262. doi:10.7556/jaoa.2019.043

4. London General Medical Council. Recommendations as to the basic medical education. 1957. Reprinted in: Simpson MA. Medical Education: A Critical Approach. London, UK: Butterworth and Company; 1972:11.

๑) 2019 American Osteopathic Association

\section{Rapid Review}

The Journal of the American Osteopathic Association considers manuscripts for rapid review when appropriate. Authors must justify their rapid review requests in their cover letters. Rapid review is reserved primarily for original contributions and reviews of high importance to the osteopathic medical profession. However, the editor in chief will assess each rapid review request on a case-by-case basis. For more information on submitting manuscripts to the JAOA, visit http://jaoa.org/ss/mssubmission.aspx. 